\title{
3 Research Square

\section{What Is the Relationship Between the Breech Presentation and Femoral Trochlear Dysplasia? An Experimental Study of the Breech Presentation Model in Neonatal Rats}

\author{
Weifeng Li \\ Hebei Medical University Third Affiliated Hospital \\ Shengjie Wang \\ Hebei Medical University Third Affiliated Hospital \\ Shiyu Tang \\ Hebei Medical University Third Affiliated Hospital \\ Zhenyue Dong \\ Hebei Medical University Third Affiliated Hospital \\ Fei Wang ( $\nabla$ doctorwfei@163.com ) \\ Hebei Medical University Third Affiliated Hospital
}

\section{Research Article}

Keywords: trochlear dysplasia, breech presentation, animal model, rat

Posted Date: October 1st, 2021

DOl: https://doi.org/10.21203/rs.3.rs-877765/v1

License: (c) (1) This work is licensed under a Creative Commons Attribution 4.0 International License.

Read Full License

Version of Record: A version of this preprint was published at BMC Musculoskeletal Disorders on January 17th, 2022. See the published version at https://doi.org/10.1186/s12891-022-05023-3. 


\section{Abstract}

Background: The relationship between breech presentation and trochlear dysplasia had been confirmed. However, the pathological process of breech-related trochlear dysplasia remain unclear. This study aimed to establish an animal model to simulate breech presentation and to analyze the pathological process of the femoral trochlea.

Materials and Methods: One hundred and twenty neonatal rats were randomly assigned into a control group and two experimental groups that were swaddled (using surgical tape) to keep the hip flexed and knees extended to simulate human breech presentation for the 5 days (short Swaddling) and the 10 days (prolonged Swaddling) of life. Gross and cross-sectional observation, histological staining measurement in two experimental time points $(5,10$ days after birth) were conducted to evaluate the morphological changes of the femoral trochlea.

Results: The incidence of trochlear dysplasia increased with the Swaddling time. Rats in the prolonged Swaddling group had the high prevalence of trochlea dysplasia (52 of 60), followed by short Swaddling group (42 of 60). Gross and cross-sectional observation showed a shallower trochlea groove in two experimental groups. Histologicalstaining measurement indicated that the trochlear sulcus angle and trochlear sulcus depth were significantly different between the experimental group and the control group since day 5 and day 10 .

Conclusion: In this model, breech presentation had a adverse influence on neonatal knees and could induce trochlear dysplasia. What is more, this study also showed that the more time in breech presentation, the more incidence of trochlear dysplasia.

\section{Introduction}

Trochlear dysplasia is a spectrum of anatomical morphological abnormalities of the distal femur [1,2]. Previous studies had showed the high incidence of trochlear dysplasia among young children and teenagers $[3,4]$. The risk factors of trochlear dysplasia include mechanical stress, genetic factor, global joint hypermobility and breech presentation, among which breech presentation is a focal point of research [5-11]. Previous study demonstrate the relationship between breech presentation and trochlear dysplasia through epidemiological methods [11]. Few reports had described the mechanism of this correlation. Also, there had been no studies indicating the pathological process of breech-related trochlear dysplasia. Since there has not been accurate method to induce breech presentation in utero, and the trials on human neonates would be controversial, animal experimental model become indispensable choice. Ren had reported that the pathogenesis of breech-related developmental dysplasia of the hip based on a newborn rat model [12]. It is well-known that the similar phenomenon of articular remodelling, such as trochlear dysplasia and hip dysplasia in children[13,14]. Knowing this made us wonder whether breech presentation could also be a predisposing factor for trochlear dysplasia. 
The purposes of the present study were to establish an simulating breech presentation animal model in neonatal rats using the surgical tape and to analyze the pathological development of the trochlear dysplasia.

\section{Materials And Methods}

The study had been approved by the Ethics Committee.

One hundred and twenty neonatal Wistar rats provided by provided by the local Animal Center were assigned into three groups. All the rats were paired sexually. The control group contains 120 knee joints, and each experimental group contains 60 knee joints. Rats in control group (CG) were left untreated. The short swaddling group (SSG) in which rats were swaddled for 5 days. The prolonged swaddling group (PSG) rats in this group underwent swaddled for 10 days. Rats in two experimental groups were swaddled with surgical tape (3 MD urapore, St.Paul, Minnesota) to keep the hips flexed and knees extended so as to simulate the human breech presentation (Fig. 1) [12]. The rats were permitted to release from the swaddling for about thirty minutes per day. All rats were fed by their mothers and cared for their normal intake.

\section{Gross observation}

120 neonatal Wistar rats were euthanized by excessive intraperitoneal injection of pentobarbital sodium $(200 \mathrm{mg} / \mathrm{kg})$ at 5 and 10 days after birth respectively $[15,16]$. Remove the skin and internal organs carefully and fix the bone tissues of distal femur in $4 \%$ paraformaldehyde overnight. the anatomic morphology of the femoral trochlea was taken to observed and recorded.

\section{Cross-sectional observation}

In order to observe the cross-sectional femoral trochlea, we chose the slice just the point of proximal to the posterior condyle. From this level, all of the femoral trochlea were sectioned axially using a scalpel [17]. Then, the cross-sectional anatomy of trochlea groove was observed in three groups.

\section{Haematoxylin and eosin (HE) staining}

\section{The specimens of knee were immersed in $4 \%$ paraformaldehyde}

$(\mathrm{pH}=7.40)$ overnight at $4^{\circ} \mathrm{C}$ and then transferred to $10 \%$ ethylene diamine tetraacetic acid (EDTA) solution about 4-5 weeks for decalcification, then, followed by alcohol and xylenes gradient dehydration. and the specimens were embedded in paraffin for subsequent tissue staining. Next, tissue slices were cut into $5-\mu \mathrm{m}$ along the femoral axis to get the transverse images of the trochlear groove. The sections were stained with HE to show the cartilage and subchondral bone $[18,19]$. Then, microscopic measurement of the morphology of femoral trochlea was performed. The pictures of representative sections were recorded by a camera. The cartilaginous trochlear sulcus angle which was defined as the angle of the deepest 
point of the trochlear connecting with the lateral trochlear cartilaginous surface and the medial trochlear cartilaginous surface, respectively. The methods applied to the measurement of cartilaginous trochlear sulcus angle, trochlear sulcus width and depth are summarized in Table 1 and Fig. 2. The measurement was performed by two blinded experienced surgeons, After an interval of 3 days, the two surgeons measured all samples again. The sulcus angle had a accuracy of $0.1^{\circ}$ measuring accuracy.The trochlear sulcus width and depth had a accuracy of $0.01 \mathrm{~mm}$ measuring accuracy. All descriptive data values were expressed by Mean \pm SD. The inter-and intraobserver reliabilities were then determined by calculating intra-class correlation coefficients (ICCs).

Table 1

Description of measurements

\section{trochlear groove morphological characteristics}

trochlear sulcus angle $(\angle A B C)$ The angle between the slopes of the medial and lateral trochlear groove. (Fig. 2)

trochlear sulcus depth (BD) Line 1 is drawn across the trochlear groove of the medial and lateral condyles. trochlear sulcus depth is the distance from the deepest portion (B) of the trochlear sulcus to line 1(D). (Fig. 2)

trochlear sulcus width (AC) The width is the distance from the

lateral condyle (A) to the medial condyle (C). (Fig. 2)

\section{Statistical analysis}

SPSS statistical software (version 22.0; SPSS, IL, USA) was performed for data analyses. The data were presented as the mean and standard deviation. The chi-square test was used to analyze differences between sexes. The normality of the distribution of values for each variable was assessed by the Shapiro-Wilks test and Dunnett's multiple-comparisons was used to evaluate between each experimental group and control group at each time point. Independent-Samples $T$ test was used for analysis of parametric data. P-value $<0.05$ was defined as the threshold for statistical significance.

\section{Results}

The inter-and intraobserver correlation coefficients was high between measurements (Table 2). All the rats in the three groups developed normally, and the weights of rats did not appear significant difference at different stages of development. 
Table 2

Intra-observer and inter-observer agreement of geometric measurements with 95\% confidence intervals(Cl)

\begin{tabular}{|lllll|}
\hline Measurement & \multicolumn{3}{l}{ Intraobserver } & \multicolumn{3}{l|}{ Interobserver } \\
\hline SSG-TSA & ICC & $95 \% \mathrm{Cl}$ & ICC & $95 \% \mathrm{Cl}$ \\
SSG-TSD & 0.785 & 0.746 to 0.825 & 0.723 & 0.687 to 0.815 \\
SSG-TSW & 0.802 & 0.707 to 0.859 & 0.768 & 0.615 to 0.804 \\
PSG-TSA & 0.853 & 0.787 to 0.939 & 0.868 & 0.805 to 0.924 \\
PSG-TSD & 0.783 & 0.696 to 0.827 & 0.702 & 0.651 to 0.785 \\
PSG-TSW & 0.786 & 0.713 to 0.838 & 0.754 & 0.726 to 0.837 \\
CG-TSA & 0.836 & 0.793 to 0.906 & 0.855 & 0.788 to 0.930 \\
CG-TSD & 0.863 & 0.814 to 0.906 & 0.828 & 0.735 to 0.908 \\
CG-TSW & 0.786 & 0.708 to 0.855 & 0.783 & 0.717 to 0.893 \\
& 0.827 & 0.735 to 0.886 & 0.850 & 0.803 to 0.936 \\
\hline $\begin{array}{l}\text { ICC, intra-class correlation coefficient; SSG,short swaddling group; TSA, trochlear sulcus angle; TSD, } \\
\text { trochlear sulcus depth; TSW, trochlear sulcus width; PSG, prolonged swaddling group; CG, control } \\
\text { group. }\end{array}$ & & \\
\hline
\end{tabular}

\section{Gross observation}

5 days after birth, the surface of the articular cartilage was smooth and the luster was bright in the short swaddling group and control group. However, Compared to control group, in the short swaddling group, the trochlear grooves were flat. 10 days after birth, the surface of the articular cartilage were no significant differences between the prolonged swaddling group and control group. The trochlear groove in prolonged Swaddling group were shallower than the control group (Fig. 3).

\section{Cross-sectional observation of trochlear morphology}

Compared to the control group, in the short swaddling group, it revealed an increase in the trochlear sulcus angle and decrease in the trochlear groove depth, the trochlear grooves were shallow. 10 days after birth, The difference of the trochlear groove between the prolonged swaddling group and control group was further aggravation. Compared to the control group, the trochlear groove showed more flattened in the prolonged swaddling group (Fig. 4).

\section{Histological analysis}

We defined the trochlear dysplasia of the two experimental groups if the trochlear sulcus angle had $5^{\circ}$ greater than the average degree of the control group. 
As we can see on the panorama of the pathological sections, the arrangement of the articular cartilage cells were orderly, and the surface of the articular cartilage was smooth in both short Swaddling group and control group at 5 days after birth. Compared with the control group, the trochlear groove was shallower in the short Swaddling group. At 10 days after birth, the difference of the trochlear groove between the prolonged Swaddling group and control group became more serious. However, the surface and arrangement of the articular cartilage cells did not appear obviously different between the two experiment groups and the control group (Fig. 5).

At 5 days after birth, the average degree of trochlear sulcus angle in control group was $130.2 \pm 3.4^{\circ}$ and the average degree of the trochlear sulcus angle in the short Swaddling group was $136.5 \pm 4.9^{\circ}$. The mean trochlear sulcus depth showed the greater difference: $0.23 \pm 0.04 \mathrm{~mm}$ in the short Swaddling group versus $0.31 \pm 0.03 \mathrm{~mm}$ in the control group (Table 3). There were significant difference between the short Swaddling group and control group at 5 days after birth (P®0.01). The mean trochlear sulcus width in control group was $1.27 \pm 0.02 \mathrm{~mm}$ and the mean trochlear sulcus width in the short Swaddling group was $1.28 \pm 0.02 \mathrm{~mm}$. No significant differences were seen in well-known measurements between the short Swaddling group and control group at 5 days after birth $(P=0.56)$. Compared with the average degree, there were 42 trochleas from 21 rats (female 14/30,male 7/30) appeared trochlear dysplasia among the total 30 rats of the short Swaddling group.

At 10 days after birth, the average degree of trochlear sulcus angle in control group was $128.5 \pm 3.4^{\circ}$, the average degree of trochlear sulcus angle in the prolonged Swaddling group was $135.8 \pm 4.6^{\circ}$. The mean trochlear sulcus depth of control group and prolonged Swaddling group was $0.42 \pm 0.02 \mathrm{~mm}$ and $0.32 \pm$ $0.03 \mathrm{~mm}$ (Table 4). Significant differences were seen in well-known measurement between the prolonged Swaddling group and control group at 10 days after birth (Pख0.01). However, the mean trochlear sulcus width in control group was $1.52 \pm 0.02 \mathrm{~mm}$ and the mean trochlear sulcus width in the short Swaddling group was $1.53 \pm 0.02 \mathrm{~mm}$. No significant differences were seen in well-known measurements between the short Swaddling group and control group at 10 days after birth $(P=0.53)$. There were 52 trochleas from 26 rats (female 14/30,male 12/30) appeared trochlear dysplasia.

Table 3. The measurements of trochlear sulcus of control group and short swaddling group $(X \pm S D)$

\begin{tabular}{|c|c|c|c|}
\hline Indexes & t swaddling grc & control groul & $p$-value* \\
\hline Mean $\mathrm{TSA}^{\circ}$ & $136.5 \pm 4.9$ & $130.2 \pm 3.4$ & $<0.01$ \\
\hline Mean TSD $(\mathrm{mm})$ & ) $0.23 \pm 0.04$ & $0.31 \pm 0.03$ & $<0.01$ \\
\hline Mean TSW(mm) & ) $1.28 \pm 0.02$ & $1.27 \pm 0.02$ & 0.56 \\
\hline
\end{tabular}

*Student's t-test.

TSA, trochlear sulcus angle; TSD, trochlear sulcus depth; TSW, trochlear sulcus width 
Table 4. The measurements of trochlear sulcus of control group and prolonged swaddling group $(X \pm$ $\mathrm{SD})$

\begin{tabular}{|lrrr|}
\hline Indexes & \multicolumn{2}{c}{ prolonged swaddling group } & \multicolumn{2}{c|}{ control group } & p-value* \\
\hline Mean TSA $^{\circ}$ & $135.8 \pm 4.6$ & $128.5 \pm 3.4$ & $<0.01$ \\
Mean TSD $(\mathrm{mm})$ & $0.32 \pm 0.03$ & $0.42 \pm 0.02$ & $<0.01$ \\
Mean TSW $(\mathrm{mm})$ & $1.53 \pm 0.02$ & $1.52 \pm 0.02$ & 0.53 \\
\hline
\end{tabular}

*Student's t-test.

TSA, trochlear sulcus angle; TSD, trochlear sulcus depth; TSW, trochlear sulcus width

Although there was a greater number of female rats than male rats appeared trochlear dysplasia at the different experimental group, the sex difference was not significant (Table 5).

Table 5

Demographic characteristics of the short swaddling group and prolonged swaddling group

\begin{tabular}{|l|}
\hline $\begin{array}{l}\text { short swaddling group prolonged swaddling group } \times 2 ~ P \\
(\mathbf{n}=\mathbf{3 0})(\mathbf{n}=\mathbf{3 0})\end{array}$ \\
\hline Gender \\
Female $14 / 30(46.67) 14 / 30(46.67) 1.620 .28$ \\
Male $7 / 30(23.33) 12 / 30(40.00)$ \\
\hline
\end{tabular}

\section{Discussion}

The key findings of the current study were that breech presentation could caused trochlear dysplasia in neonatal rats and that the incidence of trochlear dysplasia increased with the Swaddling time.

Trochlear dysplasia is characterized by a shallow, flattened

trochlear groove. They are the common knee disorder in children and adolescents with an incidence of 29 to 43 per 100000 [20]. Currently, the exact aetiology of trochlear dysplasia and the related risk factors are not consensus. However, the numerous evidence in the previous studies had revealed the correlation of trochlear dysplasia and the risk factors [21]. Breech presentation has long been reported to be a potential risk factor of trochlear dysplasia $[10,11,22]$. It is well known that the cartilaginous trochlear develops early on in gestation, between 9 and 16 weeks [23]. Given that trochlea appears well established in the prenatal period, so, it is conceivable that an intrauterine developmental process maybe the aetiology of trochlear dysplasia [24]. 
The relationship between breech presentation and trochlear dysplasia had been demonstrated through epidemiological methods [11, 24]. DeVries CA et al. recently reported that the incidence of trochlear dysplasia in breech presentation was 13.5\% [24]. Breech patients were found to have a shallower and flatter trochlear groove than those those with not breech [24]. The study by Øye CR reported that knees with extended position could bring about a 45-fold increased risk of dysplasia compared to knees with free to flex [11]. Although the relationship between breech presentation and trochlear dysplasia had been well demonstrated [11,24], there have been no experimental studies indicating the pathological process of breech position related trochlear dysplasia. Therefore, the study aimed at disclosing the pathogenesis of breech-related trochlear dysplasia based on a neonatal rat model.

Immature animal models had been frequently used to study the risk factors of trochlear dysplasia [21, 25, 26]. In the study, we fixed the rats in hip flexion and knee extension with medical tape to simulate the intrauterine breech posture that was most associated with trochlear dysplasia [11, 12]. Compared to rigid fixation, the elasticity of medical tape could allow for minor movement which was closer to the natural circumstance of intrauterine breech position [12]. It is well known that breech presentation was fixed by the time of 32 weeks of gestation [27]. In general, account for $85-90 \%$ of children born in the breech position had the knees extended [28]. So we used swaddling right similar to that in human breech presentation after birth in order that the developmental potential and morphology of the knee was the closest to intrauterine status.

In our study, a neonatal rat model was established to simulate the intrauterine breech posture. At 5 days after birth, the average degree of trochlear sulcus angle in control group was $130.2 \pm 3.4^{\circ}$ and the average degree of the trochlear sulcus angle in the short Swaddling group was $136.5 \pm 4.9^{\circ}$. The mean trochlear sulcus depth showed the greater difference: $0.23 \pm 0.04 \mathrm{~mm}$ in the short Swaddling group versus $0.31 \pm$ $0.03 \mathrm{~mm}$ in the control group. There were significant difference between the short Swaddling group and control group at 5 days after birth (Pख0.01). The mean trochlear sulcus width in control group was $1.27 \pm$ $0.02 \mathrm{~mm}$ and the mean trochlear sulcus width in the short Swaddling group was $1.28 \pm 0.02 \mathrm{~mm}$. No significant differences were seen in well-known measurements between the short Swaddling group and control group at 5 days after birth $(P=0.56)$. Compared with the average degree, there were 42 trochleas from 21 rats (female 14/30,male 7/30) appeared trochlear dysplasia among the total 30 rats of the short Swaddling group. At 10 days after birth, the average degree of trochlear sulcus angle in control group was $128.5 \pm 3.4^{\circ}$, the average degree of trochlear sulcus angle in the prolonged Swaddling group was $135.8 \pm$ $4.6^{\circ}$. The mean trochlear sulcus depth of prolonged Swaddling group and control group was $0.32 \pm 0.03$ $\mathrm{mm}$ and $0.42 \pm 0.02 \mathrm{~mm}$. Significant differences were seen in well-known measurement between the prolonged Swaddling group and control group at 10 days after birth (Pख0.01). However, the mean trochlear sulcus width in control group was $1.52 \pm 0.02 \mathrm{~mm}$ and the mean trochlear sulcus width in the short Swaddling group was $1.53 \pm 0.02 \mathrm{~mm}$. No significant differences were seen in well-known measurements between the short Swaddling group and control group at 10 days after birth $(P=0.53)$. There were 52 trochleas from 26 rats (female 14/30ロmale 12/30) appeared trochlear dysplasia. This indicated that breech presentation was associated with trochlear dysplasia, and the pathogenesis of breech-related trochlear dysplasia was a chronic process. In the study, prolonged swaddling caused a 
$16.7 \%$ increase in the prevalence of trochlear dysplasia (from $70 \%$ after short swaddling for 5 days to $86.7 \%$ after prolonged swaddling for 10 days). This finding supported that the severity of the pathological changes increases as the knees remain under this condition for a longer period. How to explain this phenomenon?

We know the unique matching relation of the patella and femoral trochlea, which is the basis of its biomechanical function [29]. Stress stimulation plays an important role in articular cartilage and subchondral bone development [7]. Researches have showed that mechanical stress is one of the most important factors affecting of cartilage and bone development [30,31]. During knee bending, the patella enters the femoral trochlear groove track and loads produce between the femoral trochlea and the patella. The mechanical loading from the patella is transmitted from the trochlear cartilage to subchondral bone. This mechanical stress stimulates the growth and remodelling of the femoral trochlea and the patella [6]. DeVries CA et al. found that breech positioning was associated with trochlear dysplasia [24], which is consisted with our finding. It is well known that the patella does not engage the trochlear groove until $30^{\circ}$ of knee flexion. So we believe that the main reason is that the mechanical stress of patella, which would result in the trochlear dysplasia. In the study, it was no significant difference of the mean trochlear sulcus width between the control group and each experimental group. The finding indicate that mechanical stress does not stimulate the growth of trochlea sulcus width in a short period after breech presentation. The female had been recognized as a risk factor of trochlear dysplasia. There was a female: male ratio of greater than 3:1 [32,33]. In our study, we found that there was a greater number of female rats than male rats appeared trochlear dysplasia at different stage of the experimental group. However, it was no significant difference in the incidence of trochlear dysplasia between the two genders. The application of swaddling might have a overwhelmed effect than that of sexual differences. The overwhelming effect of swaddling were also observed in previous studies [12,34].

There were several limitations of our study. First, we used neonatal rats to simulate breech presentation rather than establishing a real intrauterine animal model, which made it difficult to completely reflect the anatomical situation of the human. Ren et al. had endeavored to establish such a simulate breech presentation model by connecting the hindlimb skin with the chest wall through intrauterine operation. However, none of the operated pups survived the delivery [12]. Second, a continuous observation of the knee joint in animal model would have been more conclusive. However, radiological methods such as Xray or computerized tomography (CT) could not function well in such tiny knees in neonatal rats. Third, the study included the investigation of femoral groove development; However, further studies to include the morphological changes of patella could also be performed in order to get more information about breech presentation.

\section{Conclusions}

In conclusion, the present study was the first to investigate the pathological process of breech-related trochlear dysplasia. Breech presentation had a adverse influence on neonatal knees and could induce trochlear dysplasia. There was a positive linear correlation between swaddling time and the incidence of 
trochlear dysplasia. Breech-related trochlear dysplasia was a chronic process that proceeded from mildly shallower trochlear groove to trochlear dysplasia.

\section{Abbreviations}

CG: control group; SSG: short swaddling group; PSG: prolonged swaddling group; HE: Haematoxylin and eosin; EDTA: ethylene diamine tetraacetic acid; ICCs: intra-class correlation coefficients; CT: computerized tomography.

\section{Declarations}

\section{Acknowledgments}

The authors would like to thank weijing yan for revising manuscript.

\section{Authors'contributions}

WFL and FW designed the study. SJW and SYT performed the experimental work. ZYD evaluated the data. SYT draw the manuscript and made the figures and tables. WFL and SJW wrote the manuscript. All authors read and approved the final manuscript.

\section{Funding}

This study was funded by the National Natural Science Foundation of China 253 (Grant Number: 81371910).

\section{Availability of data and materials}

The detailed data and materials of this study were available from the corresponding author through emails on reasonable request.

\section{Ethics approval and consent to participate}

Institutional review board approval of the Animal Ethics Committee of the third hospital of Hebei Medical University (Number:Z2019-006-1) was obtained. All methods were carried out in accordance with relevant guidelines and regulations. The study was carried out in compliance with the ARRIVE guidelines.

\section{Consent for publication}

Not applicable.

\section{Competing interests}

The authors declare that they have no competing interests. 


\section{Author details}

${ }^{1}$ Department of joint surgery, Hebei Medical University Third Affiliated Hospital, Shijiazhaung City, Hebei Province, China.

\section{References}

1. Tscholl PM, Wanivenhaus F, Fucentese SF. Conventional radiographs and magnetic resonance imaging for the analysis of trochlear dysplasia: the inflfluence of selected levels on magnetic resonance imaging. Am J Sports Med. 2017;45:1059-1065.

2. Weber AE, Nathani A, Dines JS, et al. An algorithmic approach to the management of recurrent lateral patellar dislocation. J Bone Joint Surg Am. 2016;98:417-427.

3. Colvin AC, West RV. Patellar instability. J Bone Joint Surg Am. 2008;90(12):2751-62.

4. Clark D, Metcalfe A, Wogan C, Mandalia V, Eldridge J. Adolescent patellar instability: current concepts review. Bone Joint J. 2017;99-b(2):159-70.

5. Mittag U, Kriechbaumer A, Bartsch M, Rittweger J. Form follows function: a computational simulation exercise on bone shape forming and conservation. J Musculoskelet Neuronal Interact. 2015;15(2): 215-226.

6. Lee DY, Park YJ, Song SY, et al. Which technique is better for treating patellar dislocation? A systematic review and meta-analysis. Arthroscopy. 2018;34:3082-93.

7. Grelsamer RP, Dejour D, Gould J. The pathophysiology of patellofemoral arthritis. Orthop Clin North Am. 2008;39:269-274.

8. Parikh SN, Rajdev N, Sun Q. The growth of trochlear dysplasia during adolescence. J Pediatr Orthop. 2018;38(6):e318-e324.

9. Erden T, Aksoy DO, Ceylan HH, Kapıcioglu M, Bilsel K, Elmali N. Is femoral trochlear dysplasia related to global joint hypermobility? Journal of Orthopaedic Science. 2019;24(3):458-462.

10. Garron E, Jouve JL, Tardieu C, et al. Anatomic study of the anterior patellar groove in the fetal period [in French]. Rev Chir Orthop Reparatrice Appar Mot. 2003;89(5):407-412.

11. Øye CR, Foss OA, Holen KJ. Breech presentation is a risk factor for dysplasia of the femoral trochlea. Acta Orthop. 2016;87(1):17-21.

12. Ren $X$, Shang C, Liu TJ, Li LY, Wang Y. What is the relationship between the breech presentation and hip dysplasia? An experimental study on a rat model. Int J Clin Exp Med. 2016;9(6):10028-10037.

13. Lindstrom JR, Ponseti IV, Wenger DR. Acetabular development after reduction in congenital dislocation of the hip. J Bone Joint Surg [Am]. 1979;61-A:112-118.

14. Harris $\mathrm{NH}$. Acetabular growth potential in congenital dislocation of the hip and some factors upon which it may depend. Clin Orthop Relat Res. 1976;119:99-106.

15. Kopaladze RA. Methods for the euthanasia of experimental animals-the ethics, esthetics and personnel safety. Usp Fiziol Nauk. 2000;31(3):79-90. 
16. Zatroch KK, Knight CG, Reimer JN, Pang DS. Refinement of intraperitoneal injection of sodium pentobarbital for euthanasia in laboratory rats (Rattus norvegicus). BMC Vet Res. 2017;13(1):60.

17. Kaymaz B, Atay OA, Ergen FB et al. Development of the femoral trochlear groove in rabbits with patellar malposition. Knee Surg Sports Traumatol Arthrosc. 2013;21:1841-1848.

18. Pastoureau P, Chomel A.Methods for cartilage and subchondral bone histomorphometry. Methods Mol Med. 2004;101:79-91.

19. Ranstam J. Repeated measurements, bilateral observations and pseudoreplicates, why does it matter? Osteoarthr Cartil. 2012;20(6):473-5.

20. Fithian DC, Paxton EW, Stone ML, et al. Epidemiology and natural history of acute patellar dislocation. Am J Sports Med. 2004;32:1114-1121.

21. Yang G, Li F, Lu J, et al. The dysplastic trochlear sulcus due to the insufficient patellar stress in growing rats. BMC Musculoskelet Disord. 2019;20(1):411.

22. Salzmann GM, Dovi-Akue D, Watzig K, Sudkamp NP, Niemeyer P. Does form follow function in trochlear dysplasia? Case report of patellar instability in a below-knee amputee. Int J Surg Case Rep. 2014;5(2):91-94.

23. Yrjänä N, Kari A. The Cartilaginous Femoral Sulcus in Children with Patellar Dislocation: An Ultrasonographic Study. J Pediatr Orthoped. 1997;17:50-53.

24. DeVries CA, Hahn P, Bomar JD, Upasani VV, Pennock AT. Prevalence of trochlear dysplasia in infants evaluated for developmental dysplasia of the hip. J Child Orthop. 2021;15:298-303.

25. Li W, Wang Q, Wang F, Zhang Y, Ma L, Dong J. Femoral trochlear dysplasia after patellar dislocation in rabbits. Knee. 2013;20(6):485-9.

26. Wang S, Ji G, Yang X, et al. Femoral trochlear groove development after patellar subluxation and early reduction in growing rabbits. Knee Surg Sports Traumatol Arthrosc. 2016;24: 247-253.

27. Wilkinson JA. Etiologic factors in congenital displacement of the hip and myelodysplasia. Clin Orthop Relat Res. 1992;281:75-83.

28. Li H, Qu X, Wang Y, Dai K, Zhu Z. Morphological analysis of the knee joint in patients with hip dysplasia. Knee Surg Sports Traumatol Arthrosc. 2013;21:2081-2088.

29. Huri G, Atay OA, Ergen B, et al. Development of femoral trochlear groove in growing rabbit after patellar instability. Knee Surg Sports Traumatol Arthros. 2012;20:232-238.

30. Guevara JM, Moncayo MA, Vaca-González JJ, Gutiérrez ML, Barrera LA, Garzón-Alvarado DA. Growth plate stress distribution implications during bone development: a simple framework computational approach. Comput Meth Prog Bio. 2015;118(1):59-68.

31. Ronald PG, David D, Jason G. The Pathophysiology of Patellofemoral Arthritis. Orthop Clin N Am. 2008;39(3):269-74.

32. Colvin AC, West RV. Patellar instability. J Bone Joint Surg [Am]. 2008;90-A:2751-2762. 
33. Sillanpää P, Mattila VM, livonen T, Visuri T, Pihlajamäki H. Incidence and risk factors of acute traumatic primary patellar dislocation. Med Sci Sports Exerc. 2008;40:606-611.

34. Wang E, Liu T, Li J, Edmonds EW, Zhao Q, Zhang L, Zhao X and Wang K. Does swaddling influence developmental dysplasia of the hip? An experimental study of the traditional straight-leg swaddling model in neonatal rats. J Bone Joint Surg Am. 2012;94: 1071-1077.

\section{Figures}

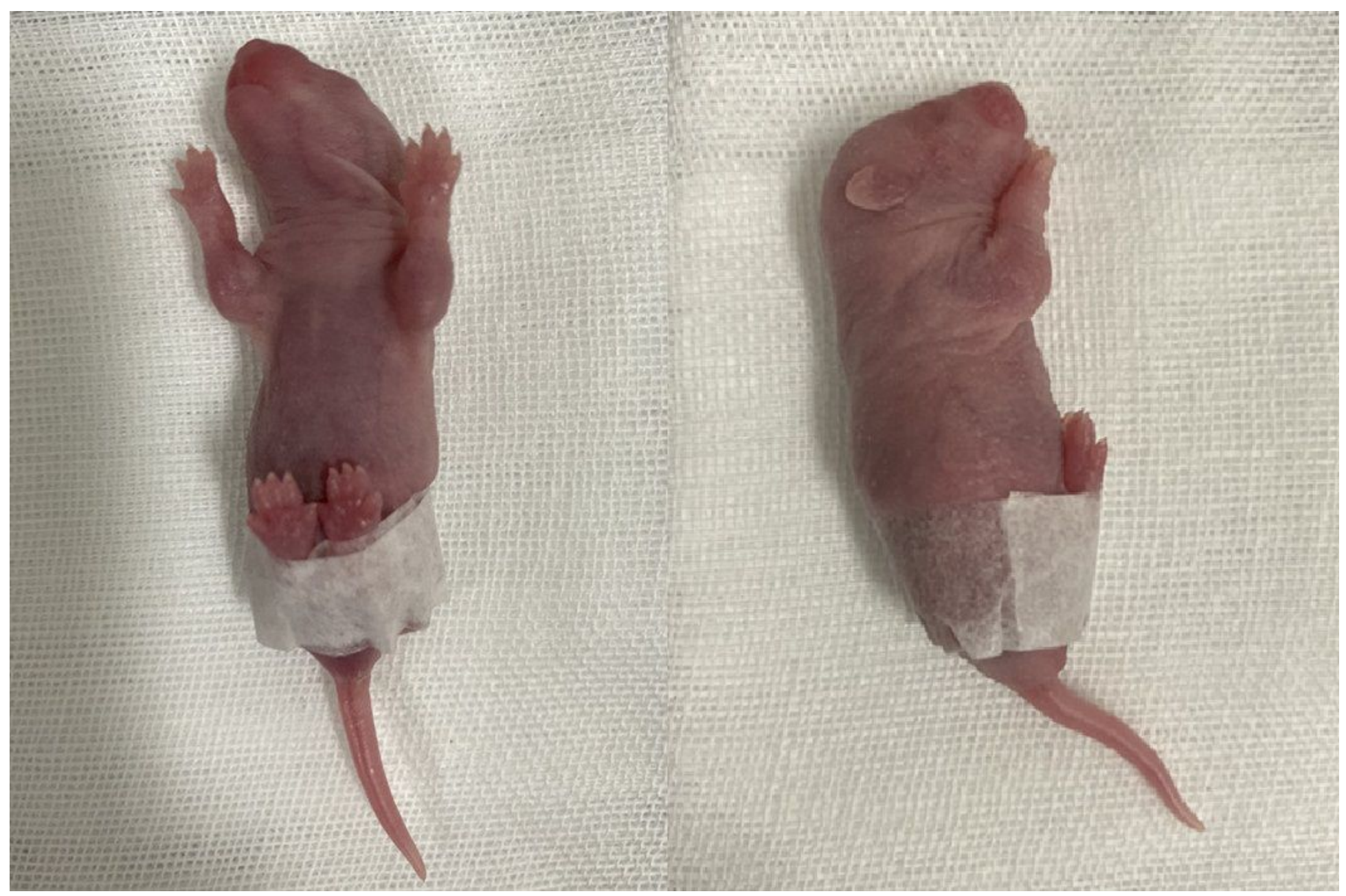

\section{Figure 1}

The swaddling model of frank-breech presentation. The rat was swaddled with surgical tape to keep the hip flexed and knees extended so as to simulate human frank-breech presentation. 


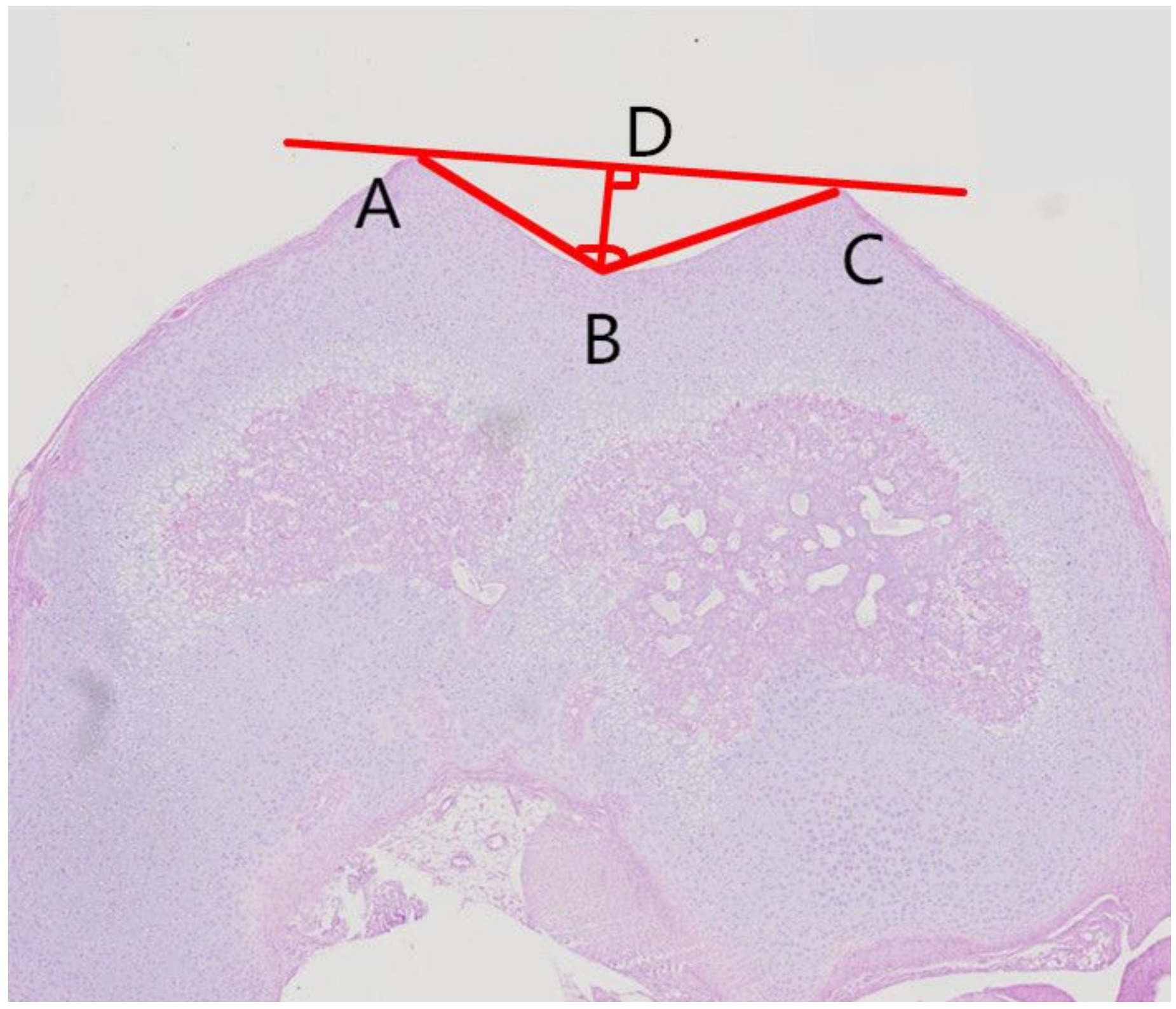

\section{Figure 2}

The angle $\angle A B C$ is defined as the trochlear sulcus angle, $A C$ is defined as the trochlear sulcus width; $B D$ is defined as the trochlear sulcus depth. 

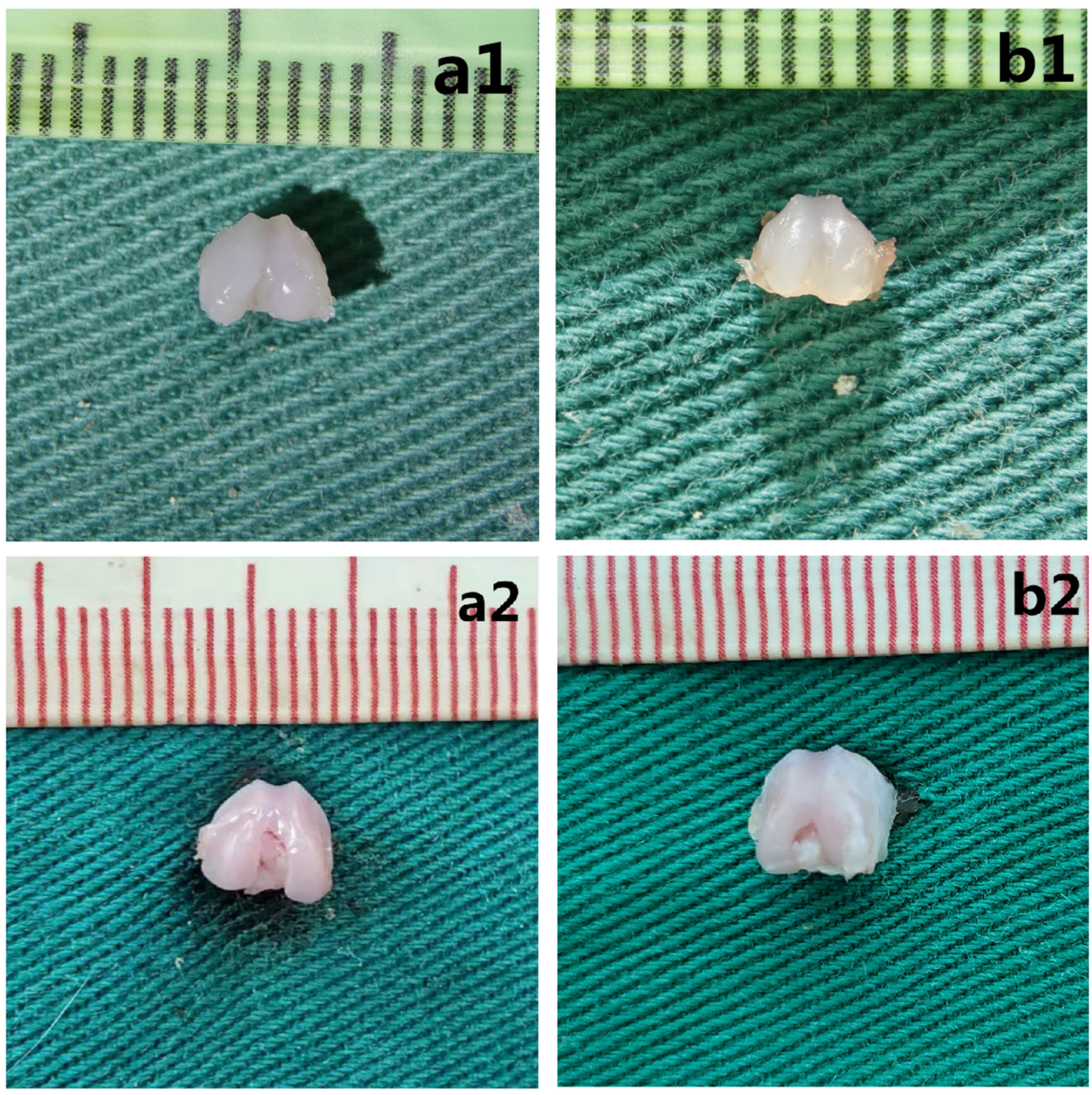

Figure 3

Gross anatomy of the femoral trochlea: a1 5 days of the control group, b1 5 days of the short Swaddling group; a2 10 days of the control group and b2 10 days of the prolonged Swaddling group. 

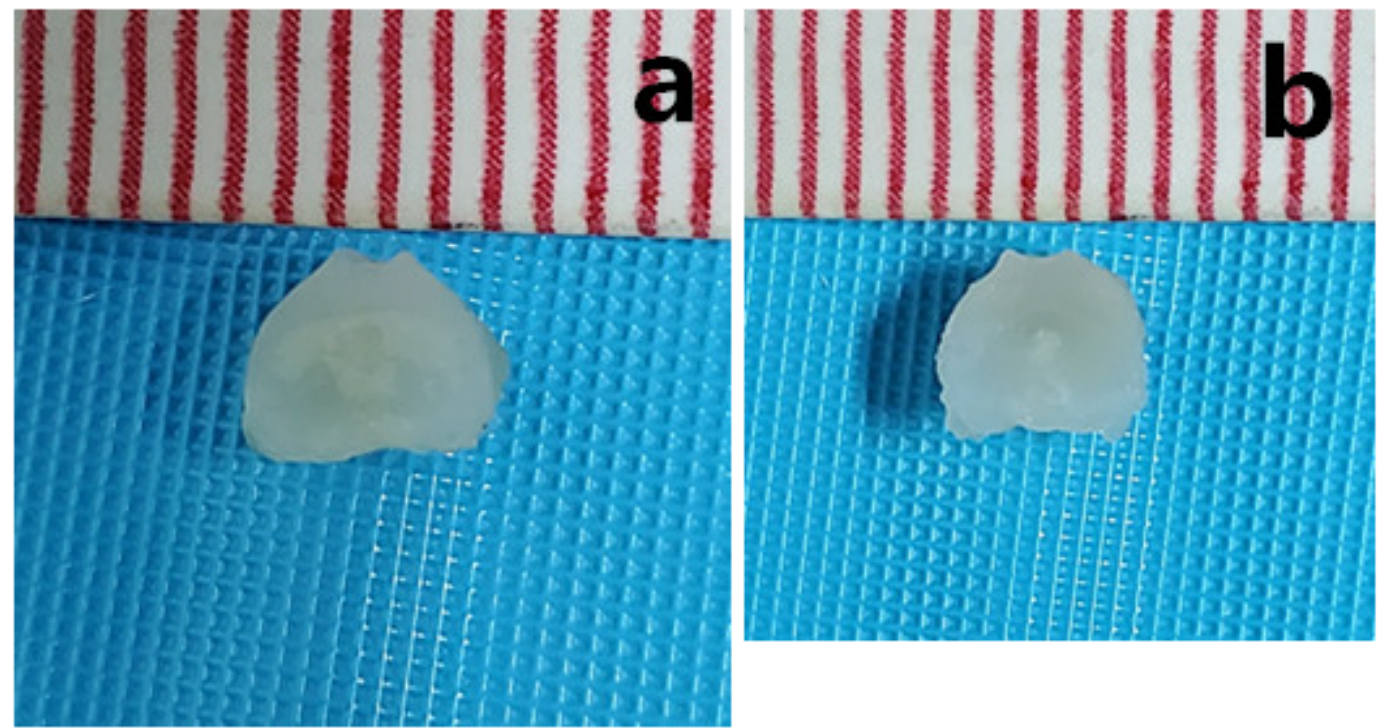

Figure 4

Cross-sectional observation: axial image of the femoral trochlea: a 10 days of the control group; $b 10$ days of the prolonged Swaddling group. 

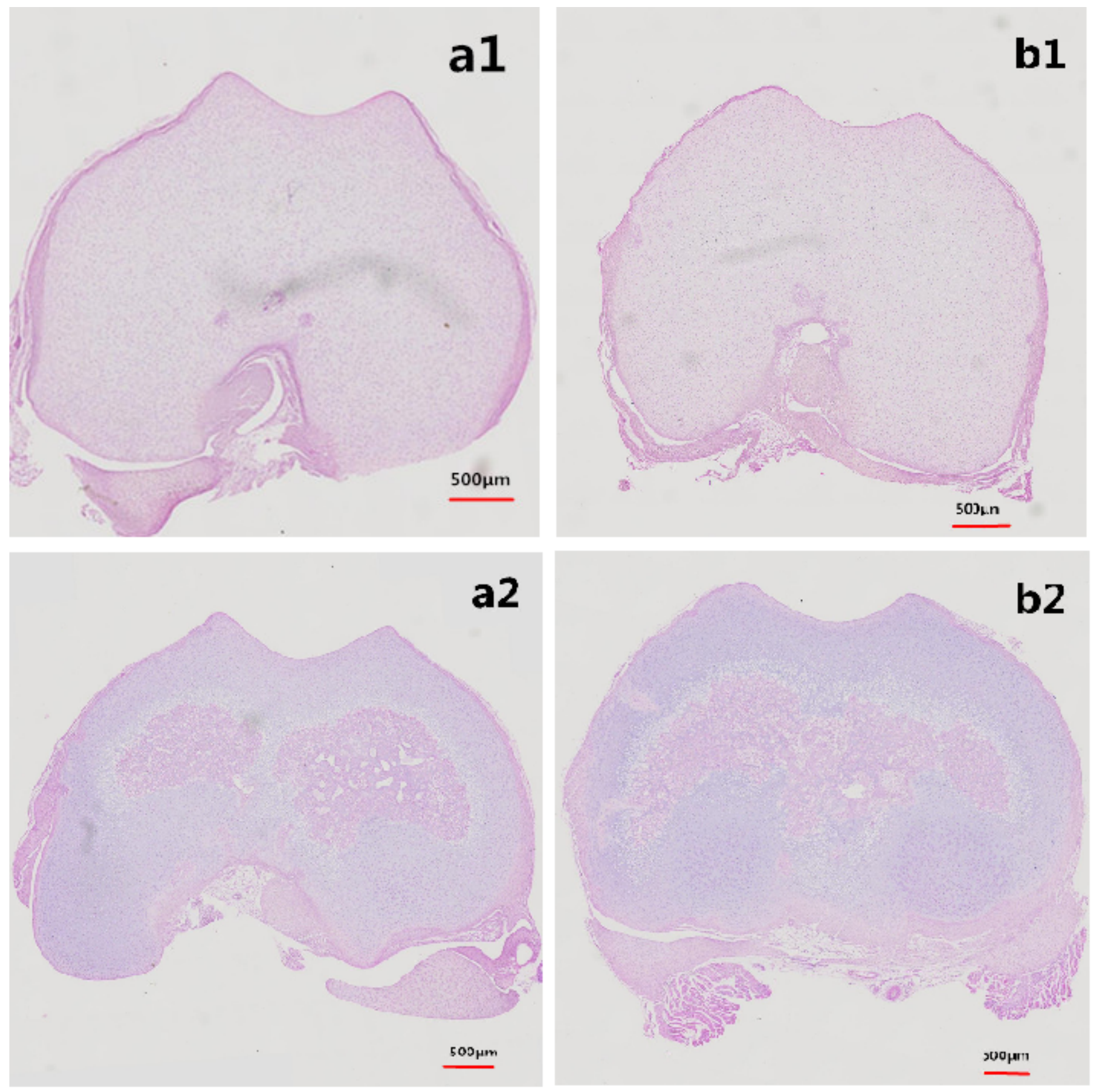

Figure 5

Microscopic view of femoral trochlear by HE staining: a1 5 days of the control group, b1 5 days of the short Swaddling group; a2 10 days of the control group and b2 10 days of the prolonged Swaddling group. 